\title{
Spectral Analysis of the Primary Flight Focal Plane Arrays for the Thermal Infrared Sensor
}

NASA Goddard Space Flight Center ${ }^{1}$

Rochester Institute of Technology²

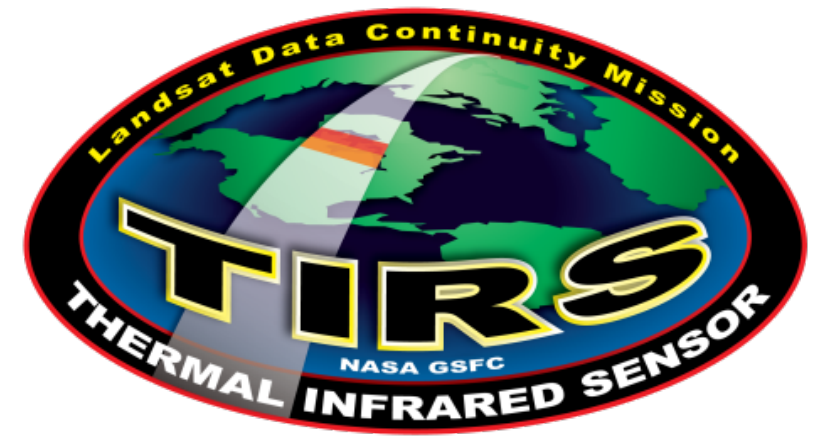

Matthew Montanaro ${ }^{1}$

Dennis C. Reuter ${ }^{1}$

Brian L. Markham ${ }^{1}$

Kurtis J. Thome ${ }^{1}$

Allen W. Lunsford ${ }^{1}$

Murzy D. Jhabvala ${ }^{1}$

Scott O. Rohrbach ${ }^{1}$

Aaron D. Gerace ${ }^{2}$ 




\section{Spectral Filter Mask}
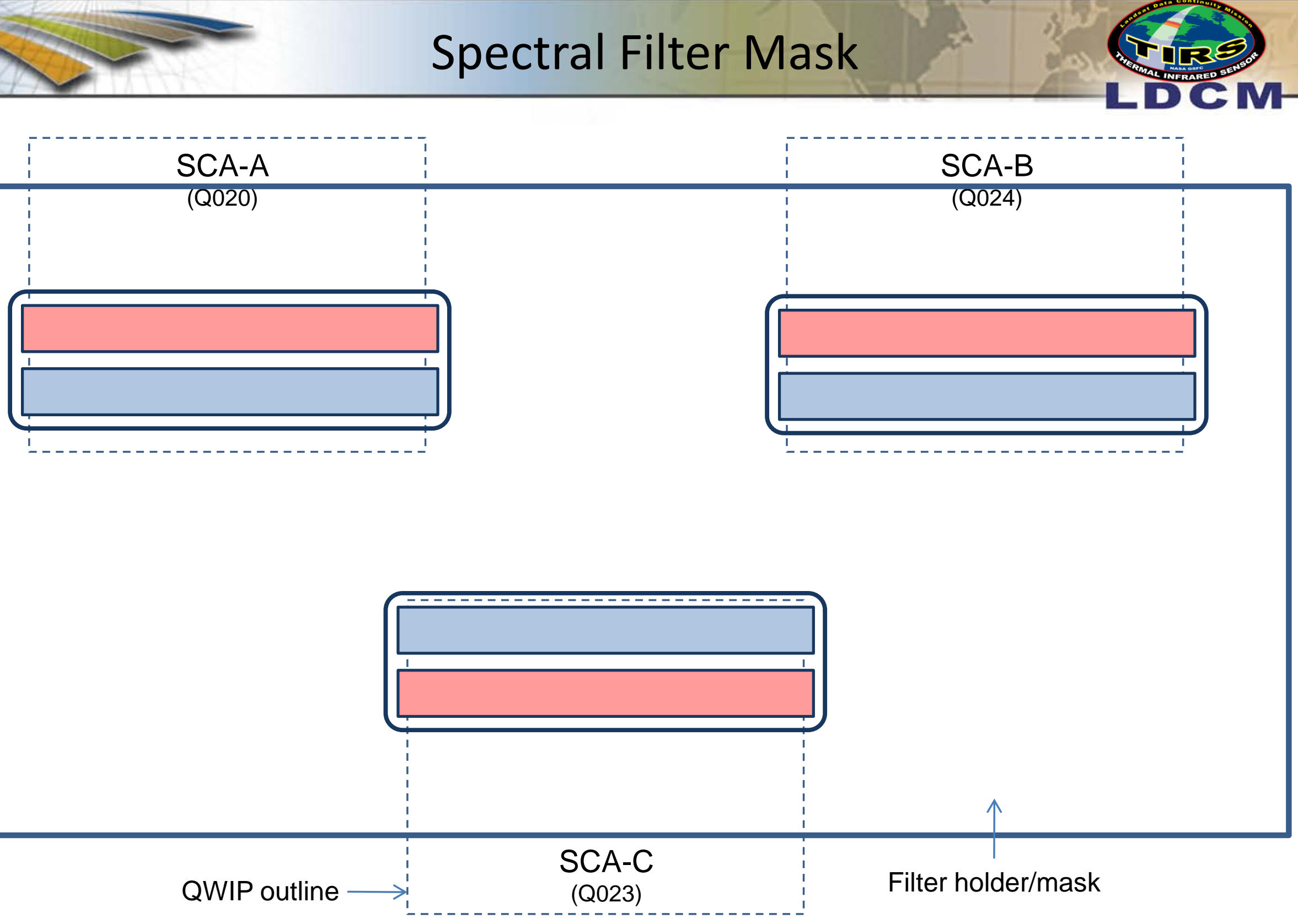






Study 1: Uniform Scenes 



\section{Uniform Scene}

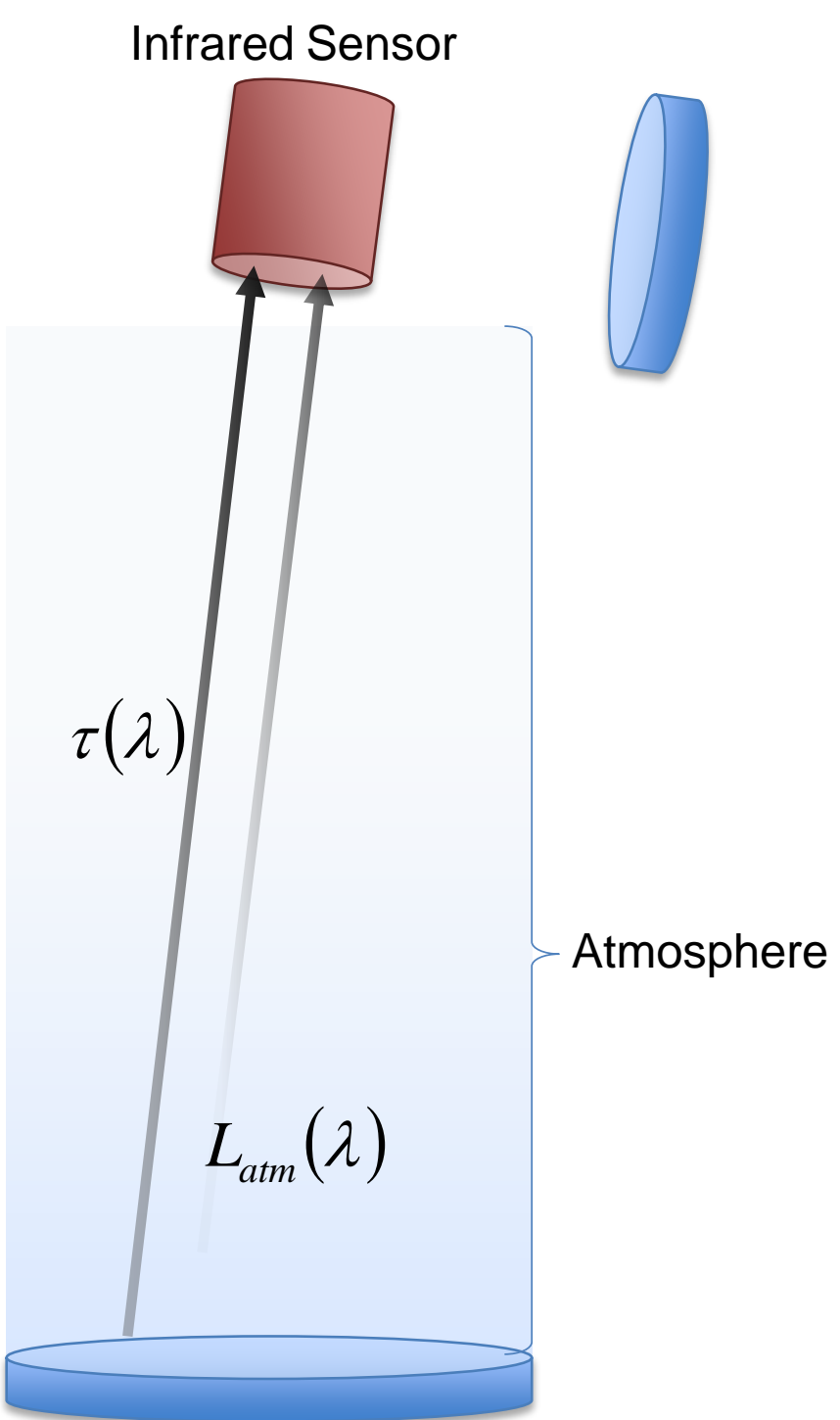

Uniform Blackbody $B(T, \lambda)$

$$
S_{i}=\frac{\int\left[B(T, \lambda) \cdot \tau_{a t m}(\lambda)+L_{a t m}(\lambda)\right] \cdot R_{i}(\lambda) \cdot d \lambda}{\int R_{i}(\lambda) \cdot d \lambda}
$$

- Same calibration on uniform scene through atmosphere

- Will not return same brightness temperature due to atmospheric terms
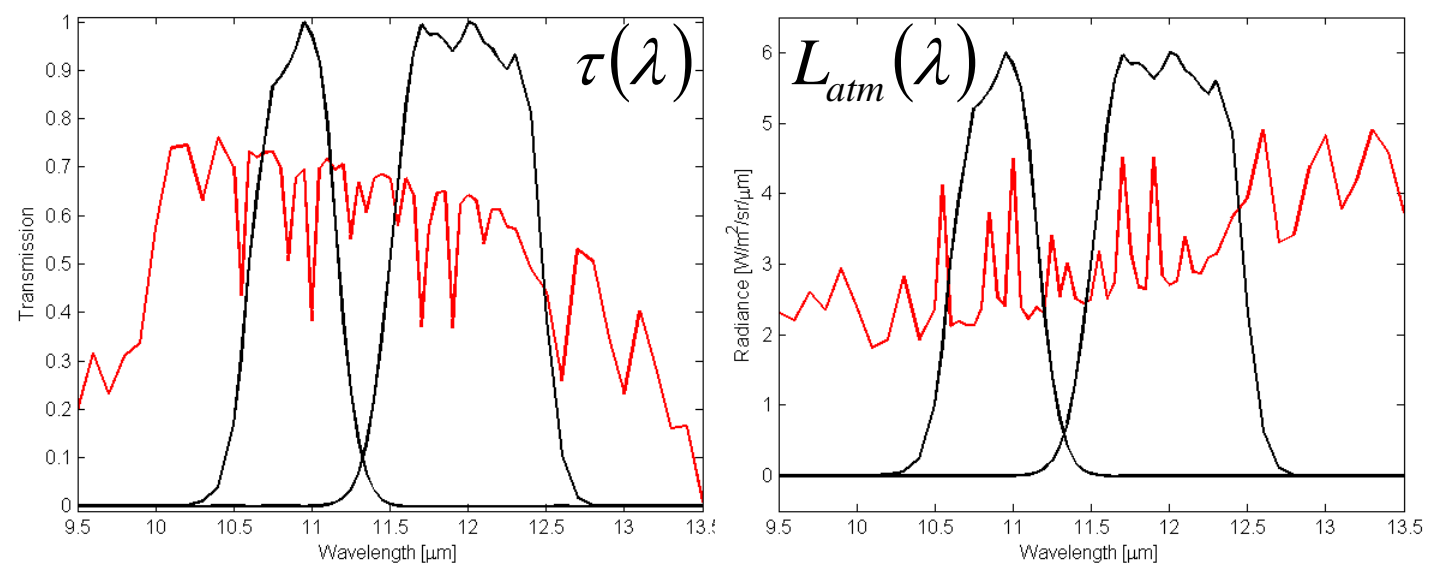

-What is the residual striping effect due to the atmosphere when viewing a uniform blackbody? 


\section{Study 1 Procedure}

$$
S_{i}=\frac{\int\left[B(T, \lambda) \cdot \tau_{a t m}(\lambda)+L_{a t m}(\lambda)\right] \cdot R_{i}(\lambda) \cdot d \lambda}{\int R_{i}(\lambda) \cdot d \lambda}
$$

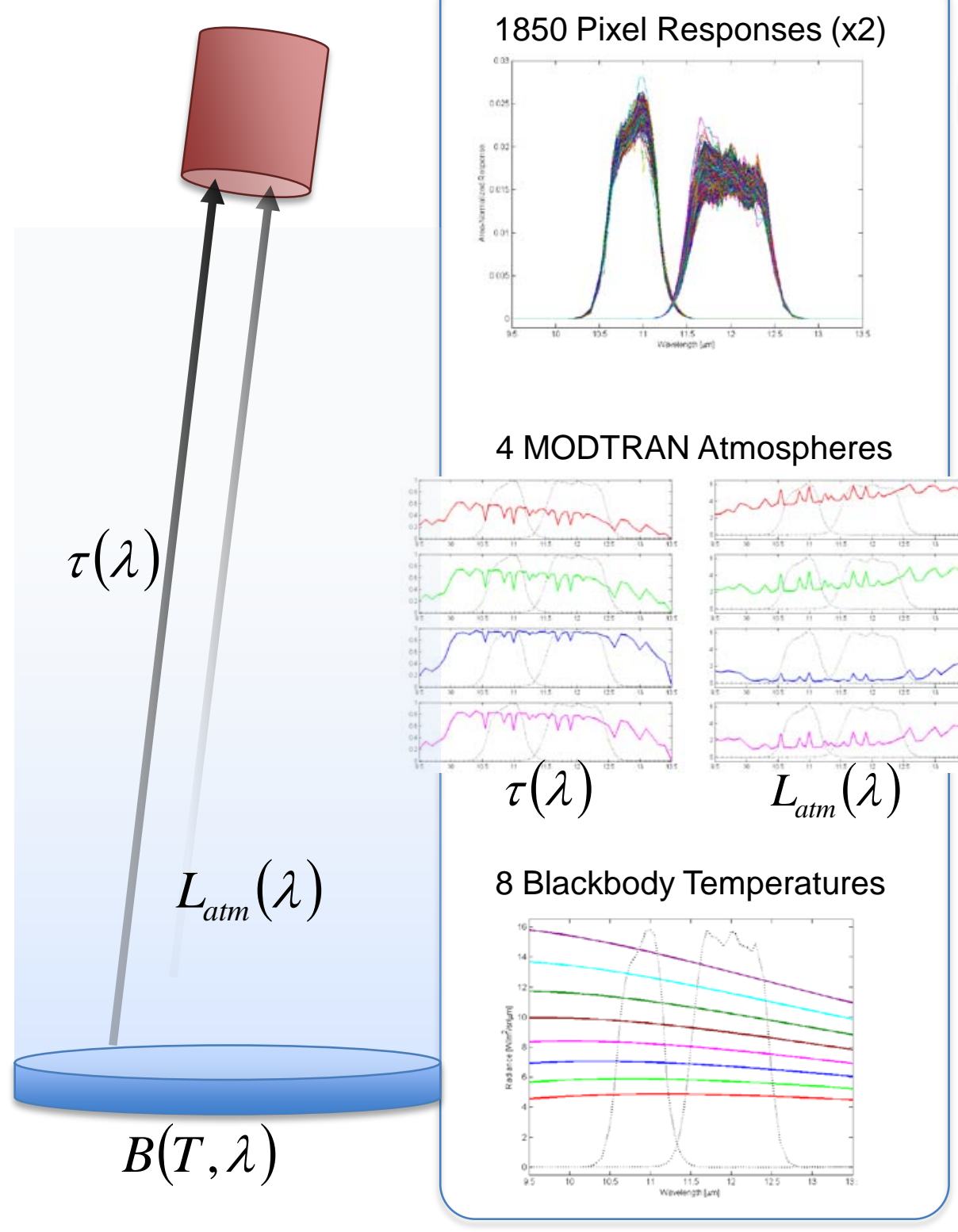

4 MODTRAN Atmospheres

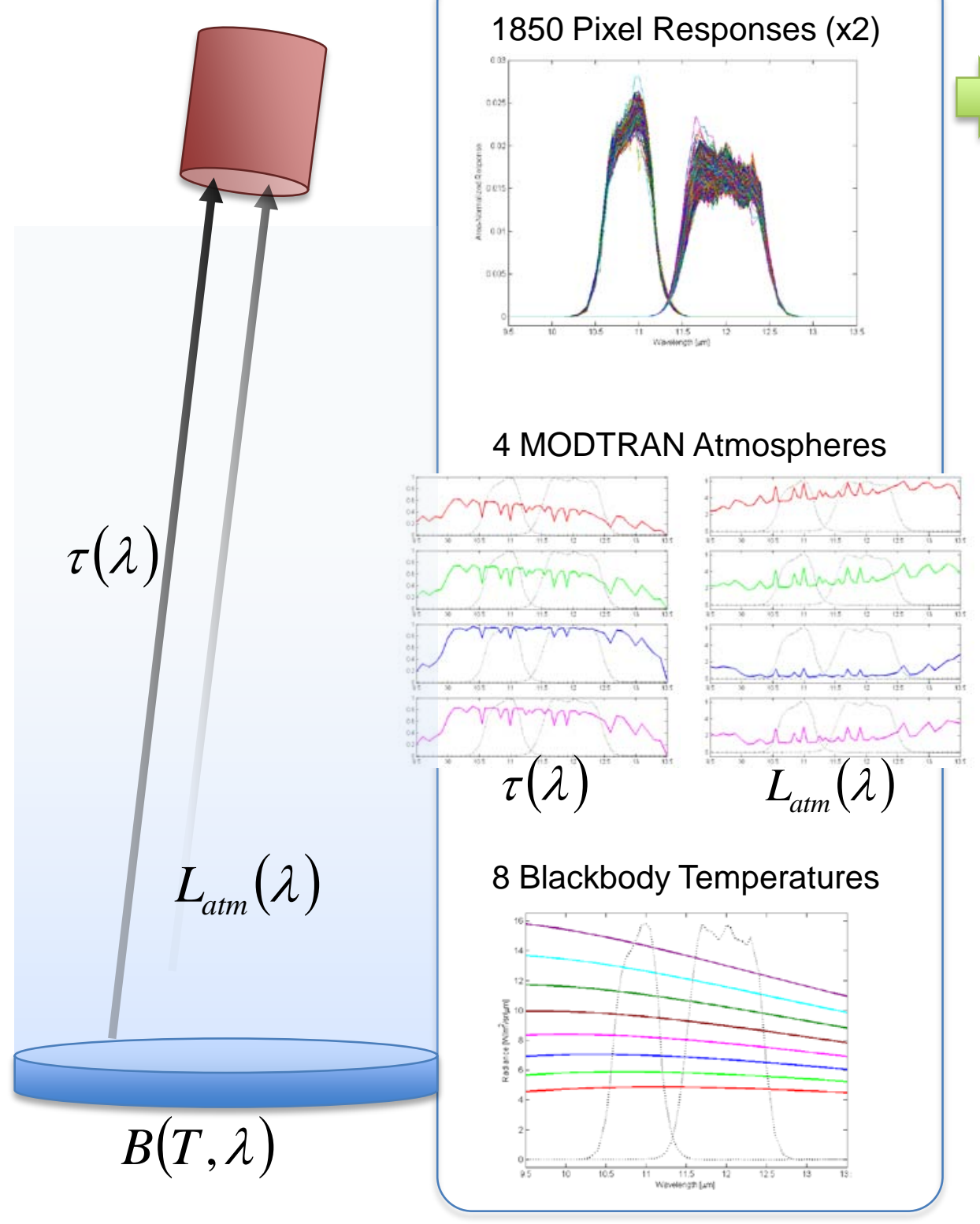

$L_{a t m}(\lambda)$

8 Blackbody Temperatures

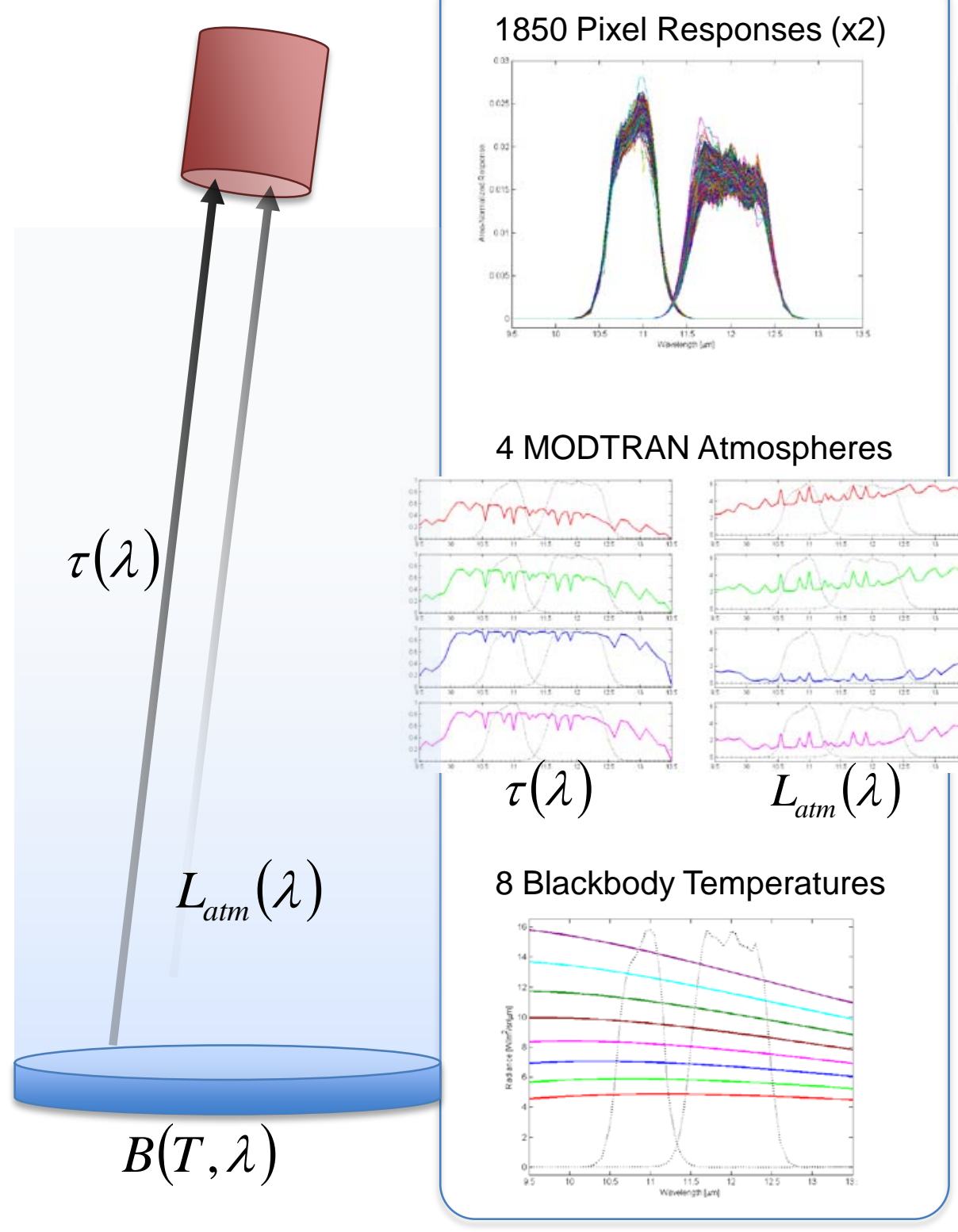

Residual Striping?
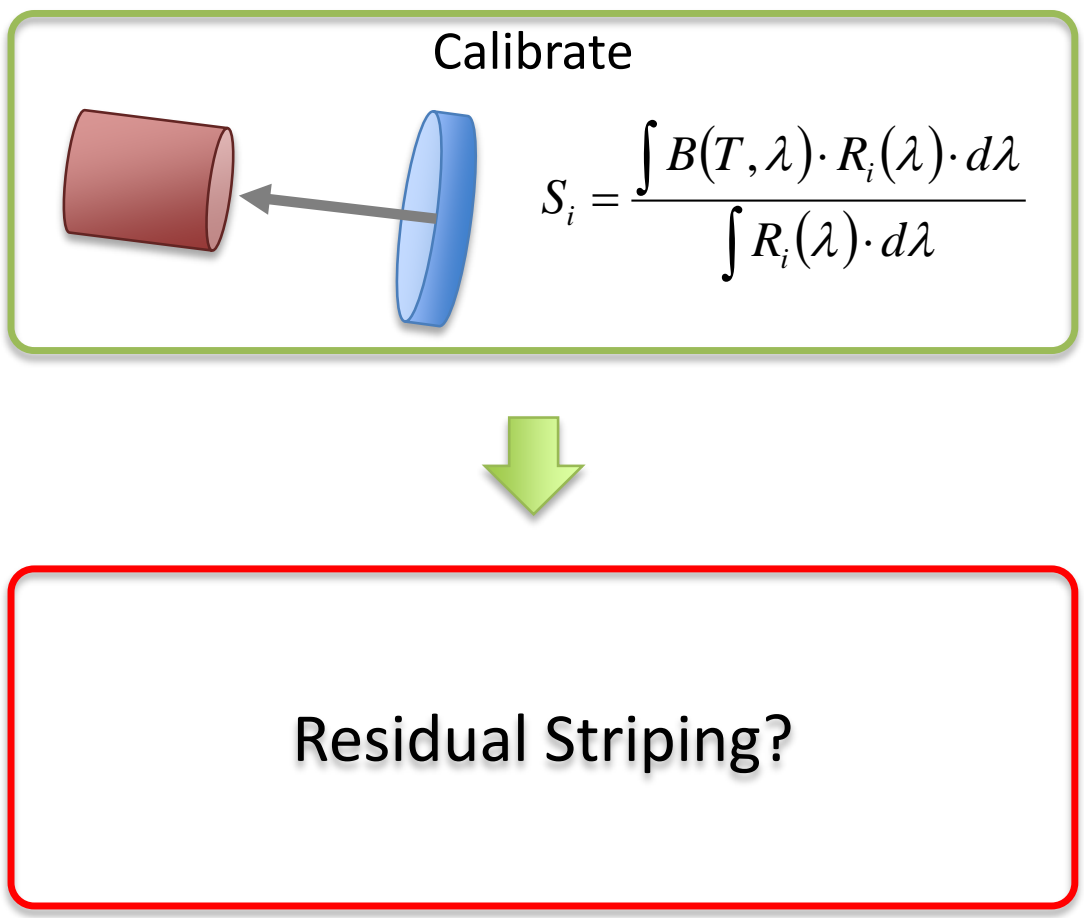


\section{Study 1 Results - 10.8 Band}

280 K Blackbody Target
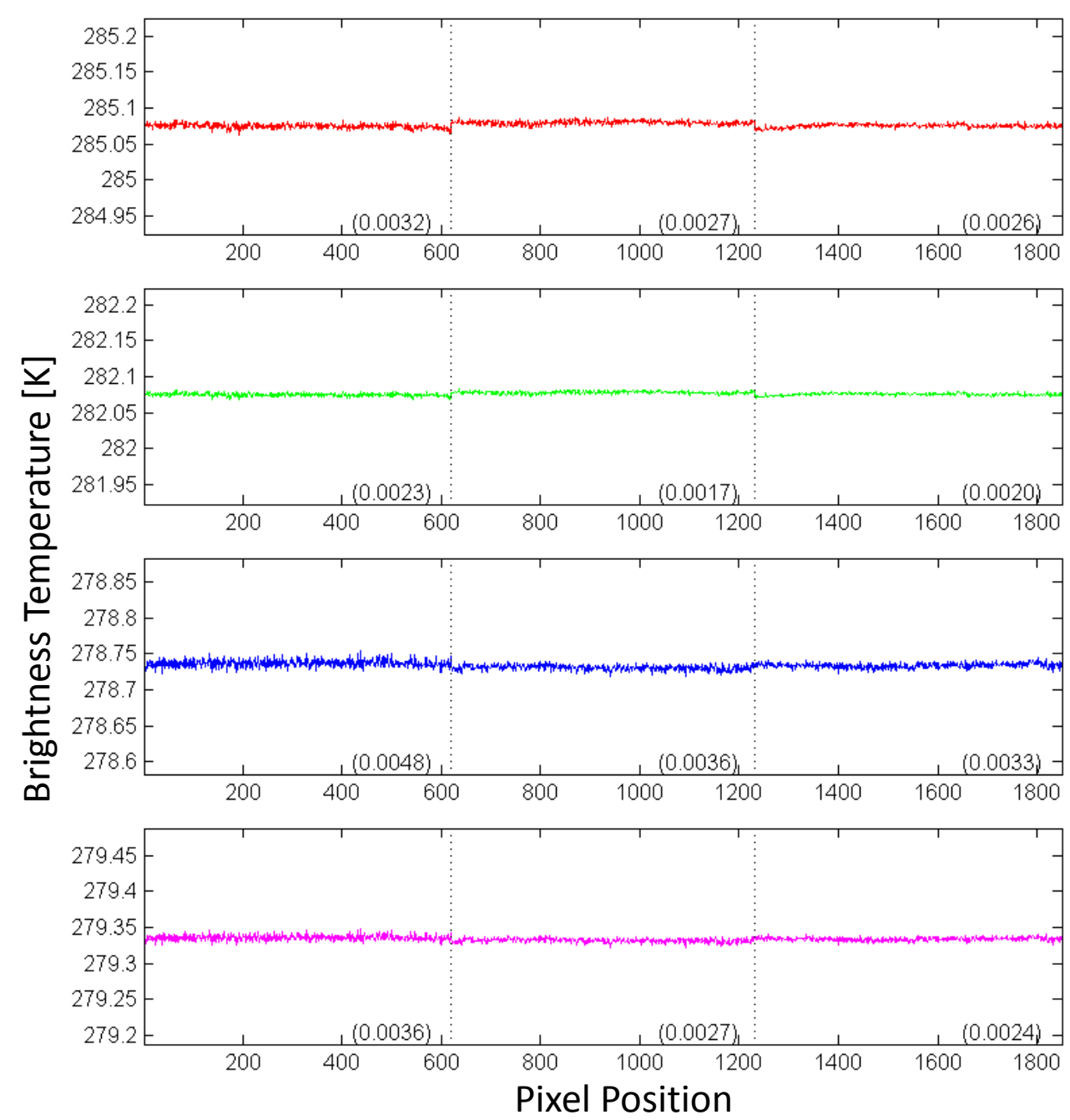

MODTRAN Standard Atmospheres:

Tropical

Mid-Latitude Summer
330 K Blackbody Target
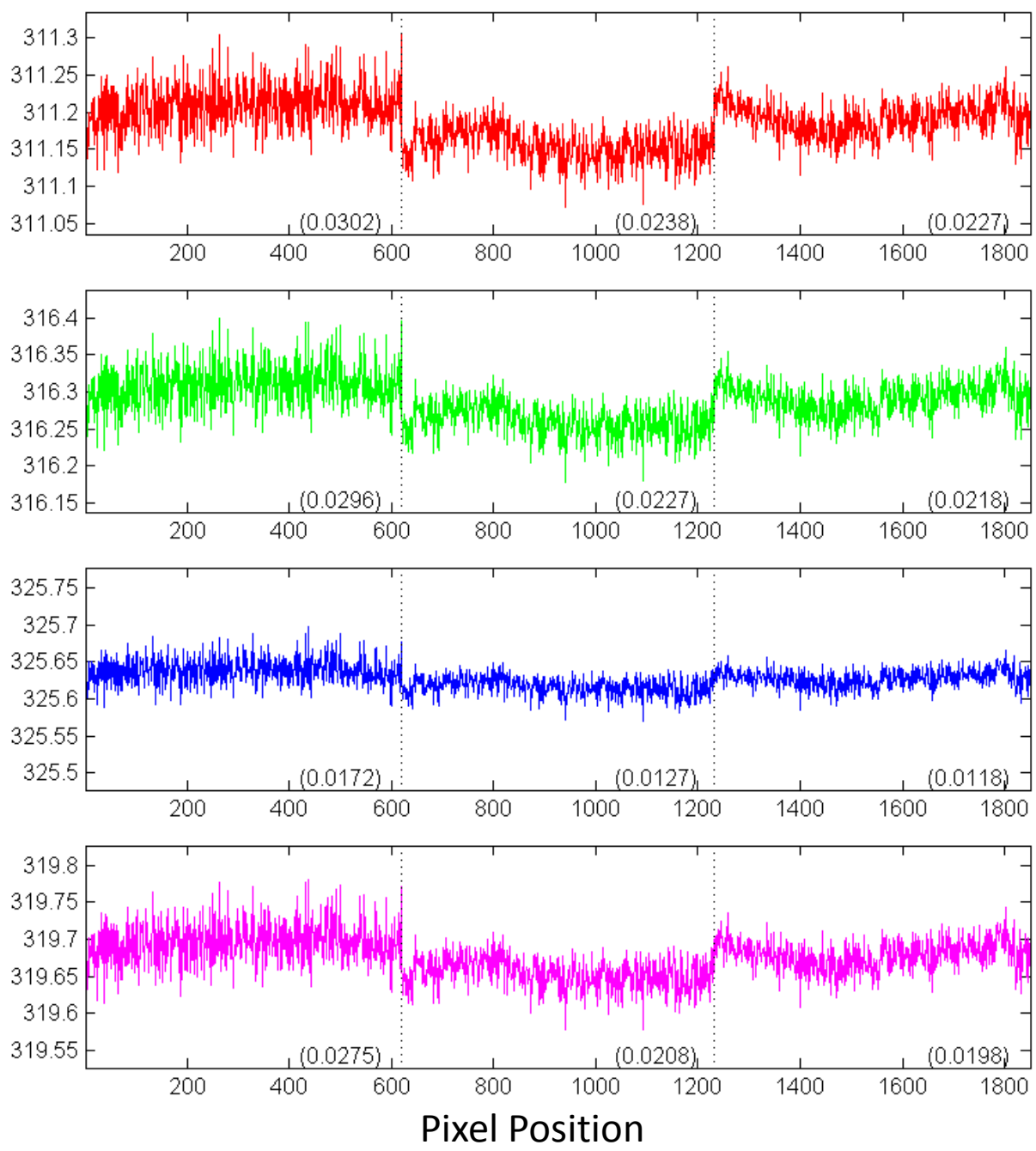

Mid-Latitude Winter Sub-Arctic Summer 


\section{Study 1 Observations}

Striping even after radiometric calibration of the detectors

-Calibration based on a smooth blackbody radiance

- Atmospheric spectral variations = different integrated signal for a particular detector.

Minimum for mid-latitude winter atmospheres and maximum for tropical atmospheres

- Tropical atmosphere: higher transmission and path radiance effects = magnify striping artifacts

Striping minimized for temperatures of $270-280 \mathrm{~K}$ -contrast between the target temperature and effective atmospheric temperature.

- transmission losses compensated by path radiance

Striping is generally greater in $\mathbf{1 2 . 0}$ micron channel

- wider bandwidth more susceptible to spectral variations

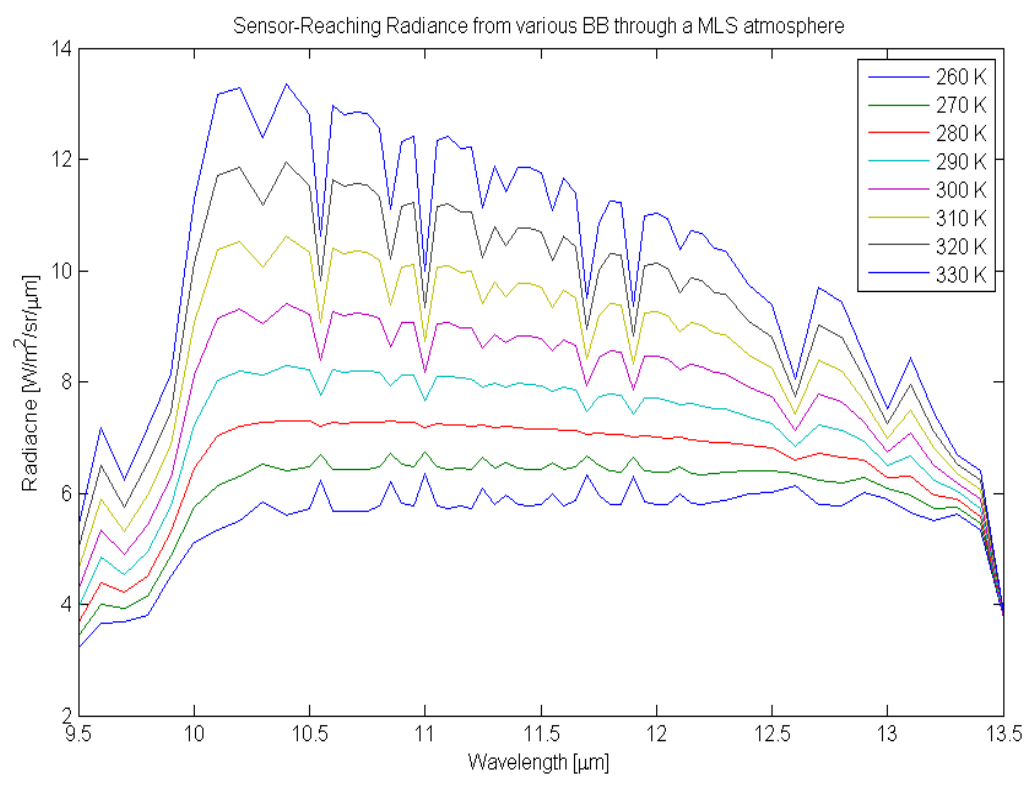

The residual artifacts expected to be small (max standard deviations $35 \mathrm{mK}$ and $57 \mathrm{mK}$ ) 
Study 2: Realistic Scenes 


\section{Realistic Scene}

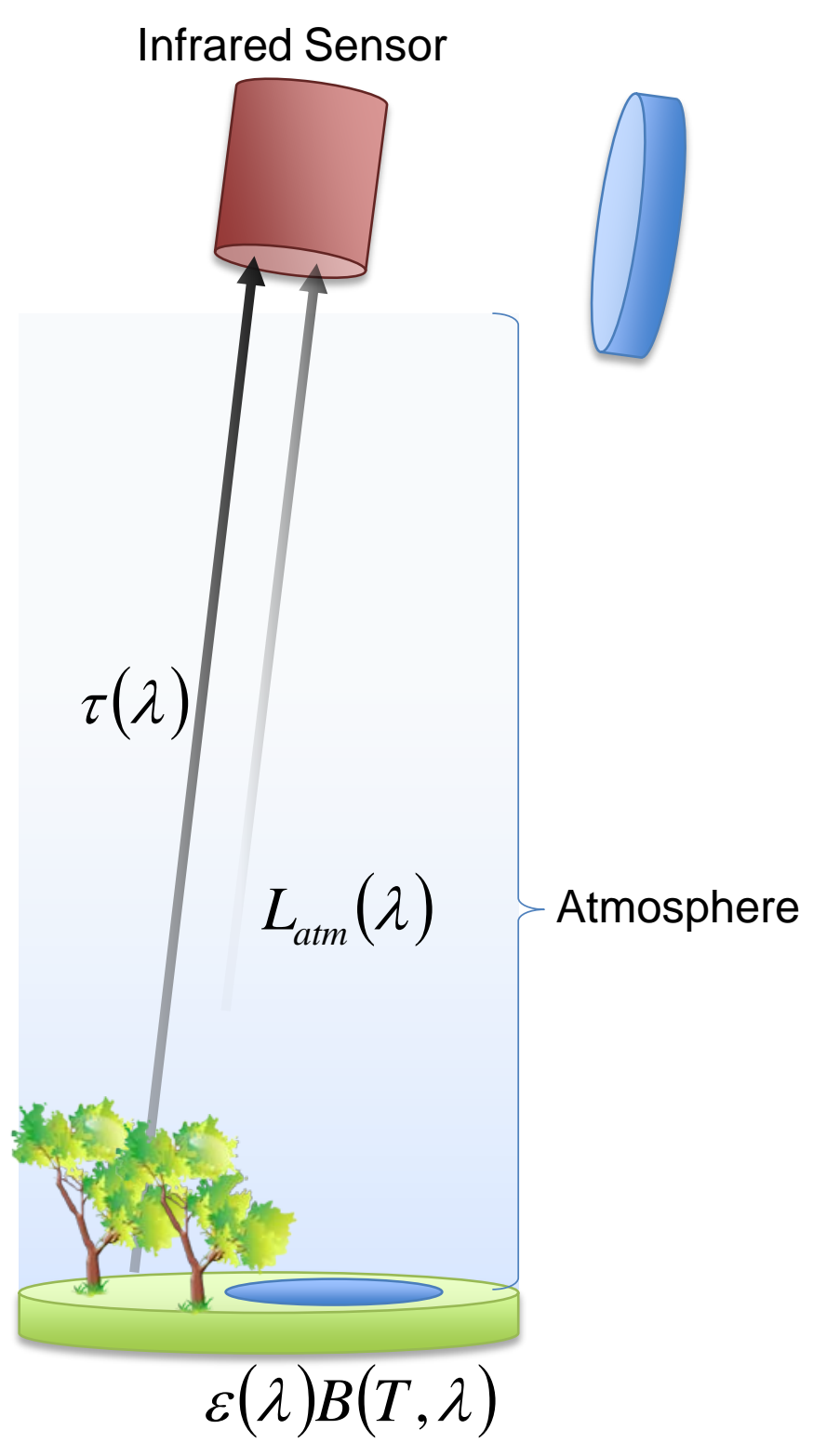

$S_{i}=\frac{\int\left[\varepsilon(\lambda) B(T, \lambda) \cdot \tau_{a t m}(\lambda)+L_{a t m}(\lambda)\right] \cdot R_{i}(\lambda) \cdot d \lambda}{\int R_{i}(\lambda) \cdot d \lambda}$

- Replace uniform scene with realistic scene

- Various emissivities

- Group pixel responses into classes

- Representative spectral shapes

How do different band shapes affect the brightness temperature in a realistic scene? 


\section{DIRSIG Lake Tahoe Image}

DIRSIG Thermal Radiance image [W/m2/sr/um]

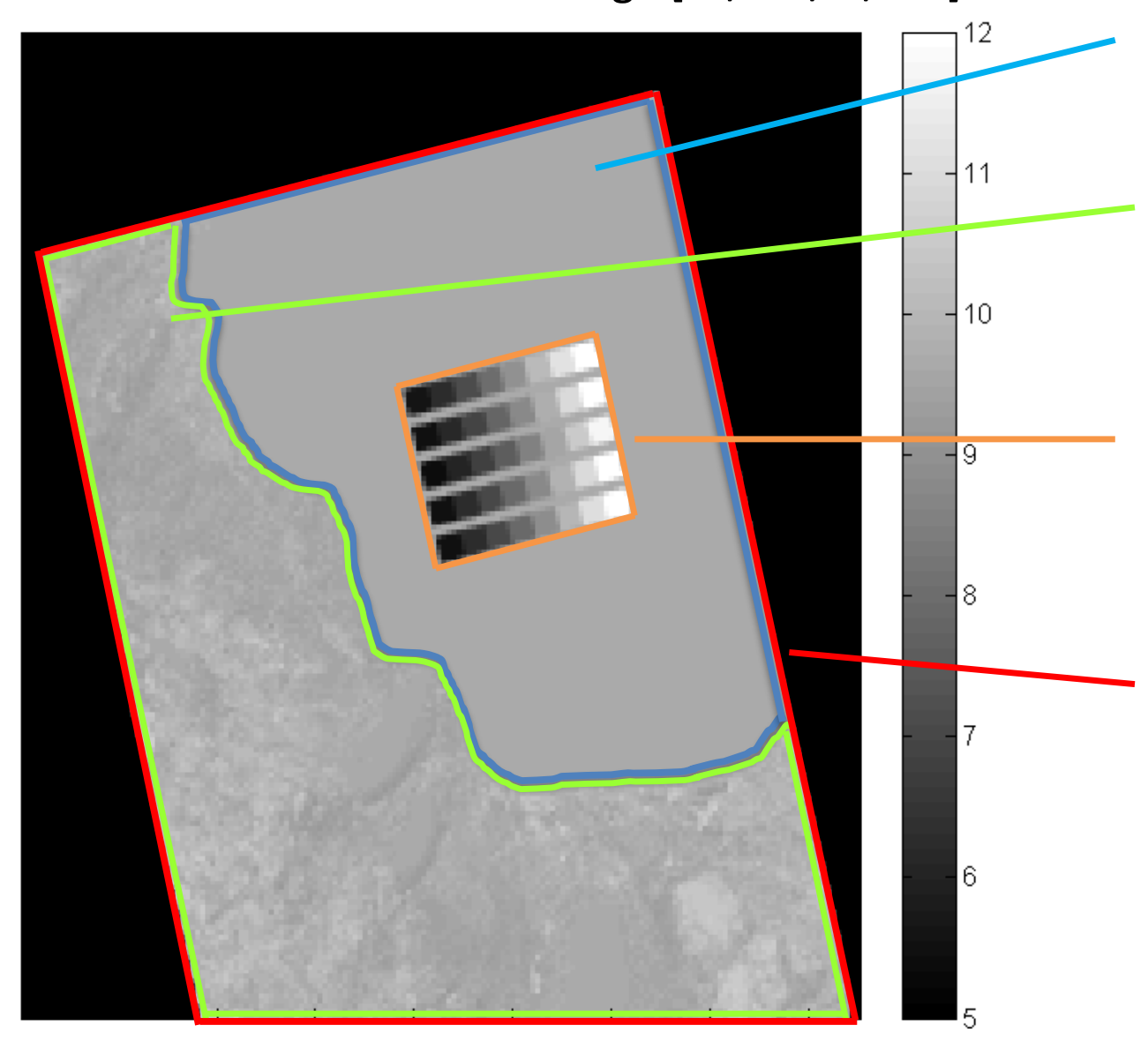

Lake:

- Water, sea-level

Terrain:

- Various land cover types

- Relief via DEM

Panels:

- Various emissivities

- Various temperatures

Atmosphere:

- Uniform atmosphere for entire scene

- Atm. terms generated via MODTRAN 


\section{DIRSIG Lake Tahoe Image}

DIRSIG Thermal Radiance image [W/m2/sr/um]

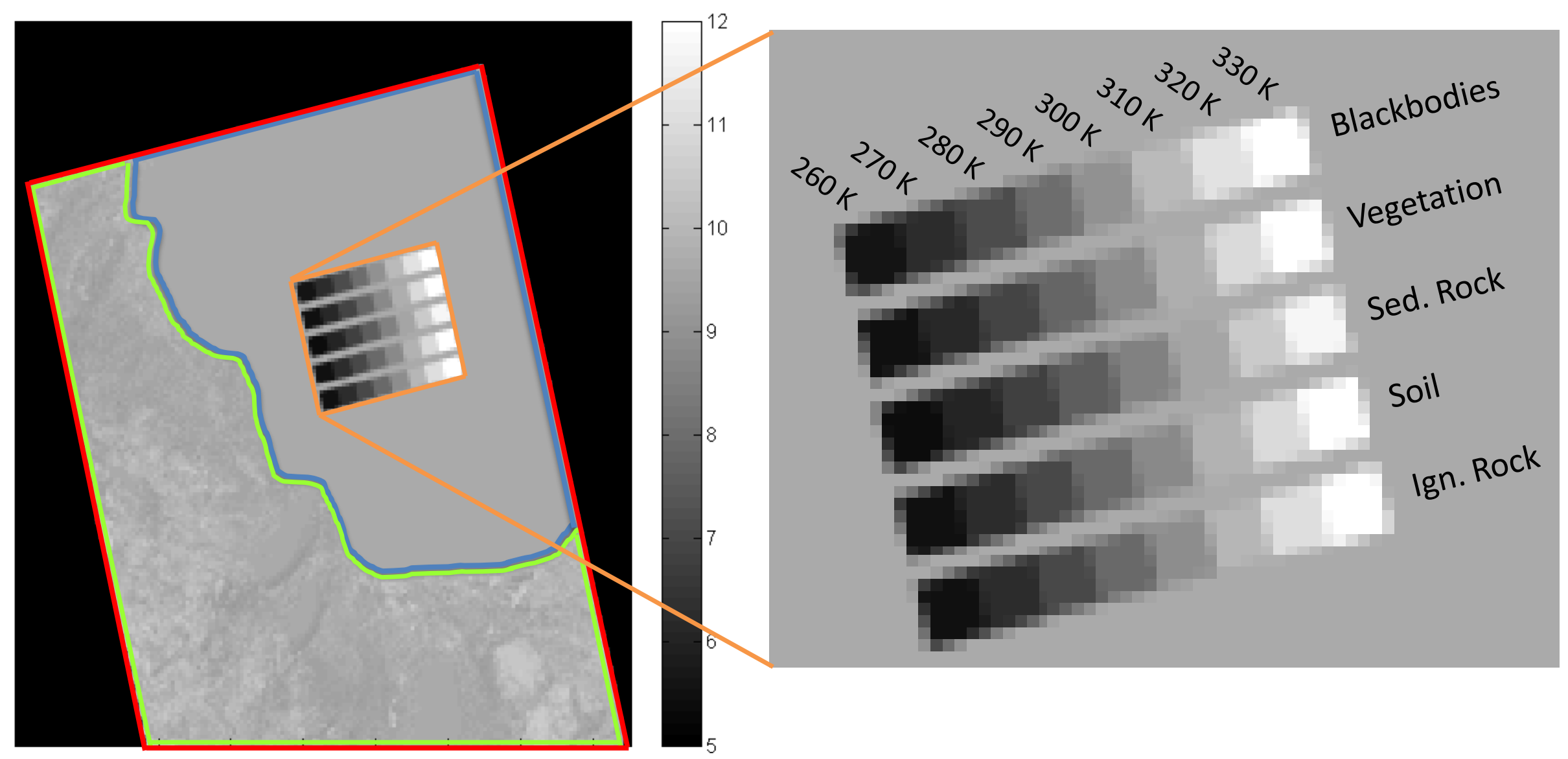






\section{Study 2 Observations}

Temperature differences generally greater in 12.0 micron channel

- wider bandwidth more susceptible to spectral variations

- greater variation in 12 micron band shapes

Temperature differences greatest for tropical atmosphere - higher transmission and path radiance effects = increased spectral variation

Material type affects temperature differences (very subtle)

- lower emissivity materials less susceptible to band shape

- lower emissivity = more reflected atmosphere = less contrast between ground and atm .

Results consistent with previous study

Band shape differences expected to be $0.1 \%$ and a $0.2 \%$ 






\section{Atmosphere Types}

MODTRAN Standard Atmospheres:

- Tropical

Atmospheric Transmission

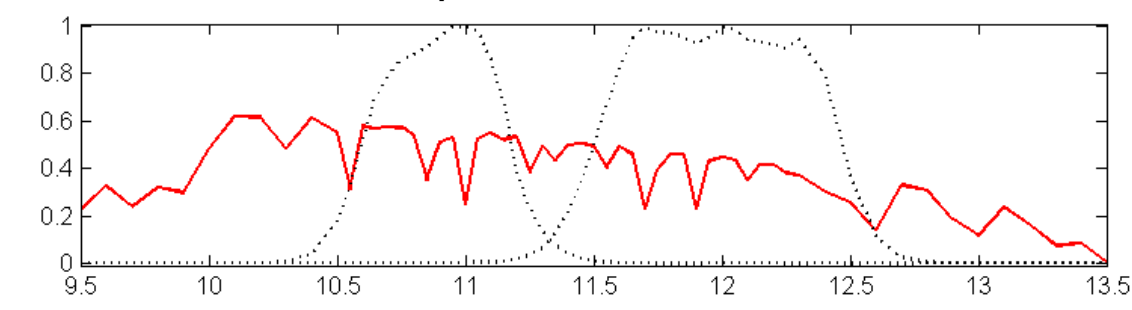

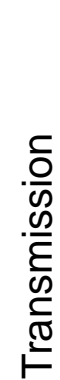
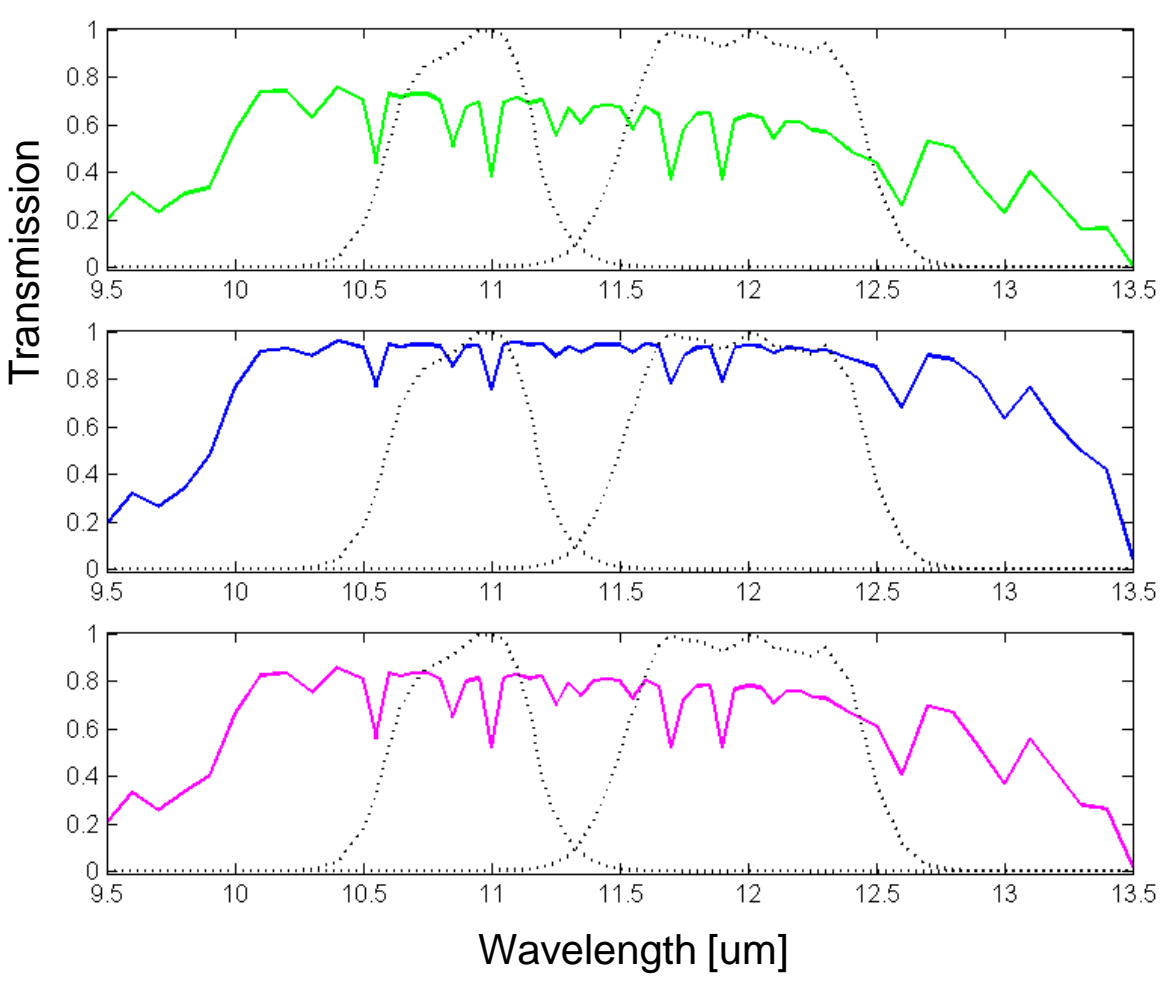

Mid-Latitude Winter

Sub-Arctic Summer

Atmospheric Path Radiance

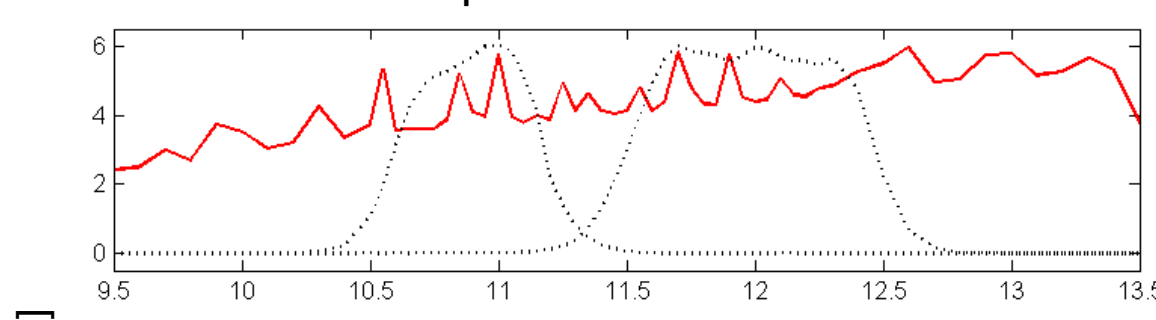

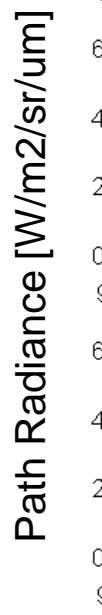
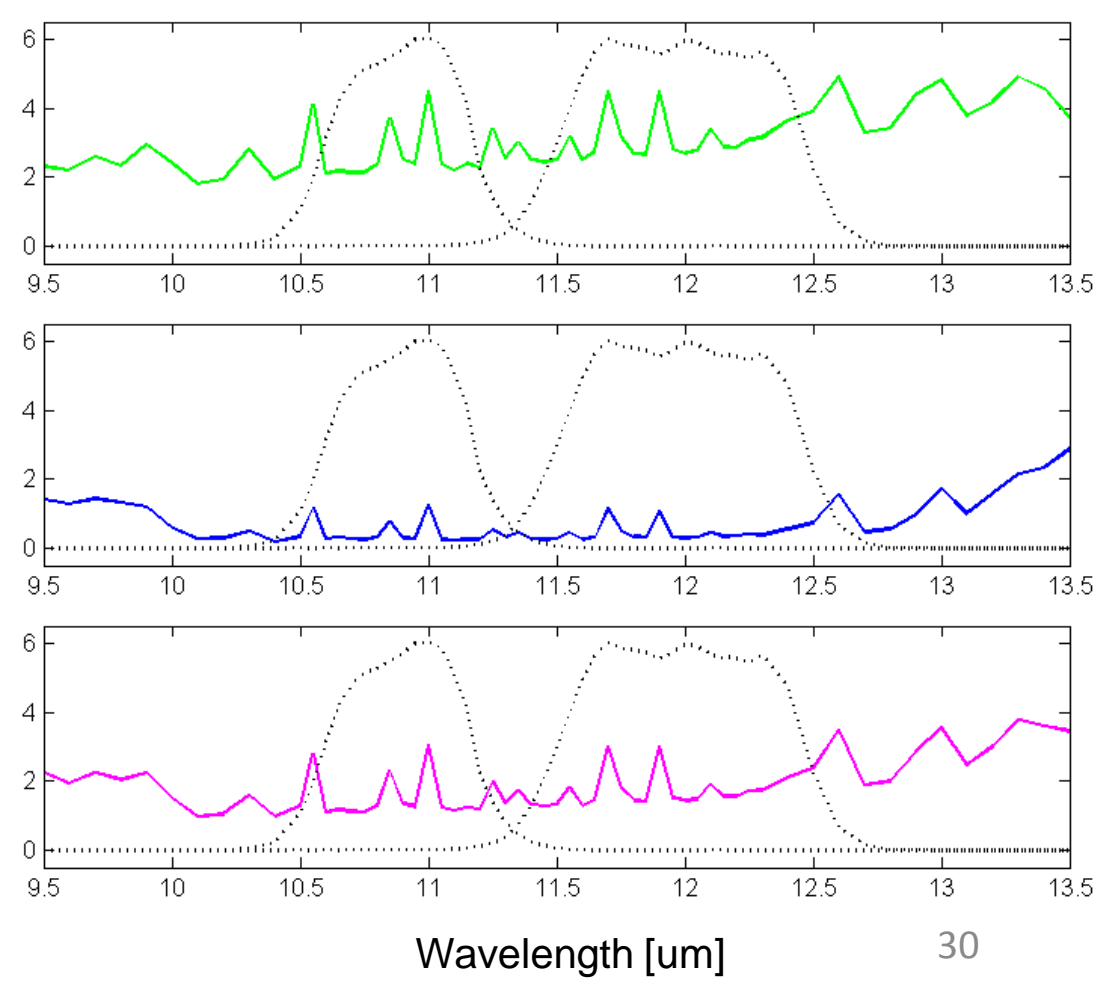




